\title{
Farmacoterapia atual das náuseas e vômitos induzidos por antineoplásicos
}

\author{
Current pharmacotherapy of antineoplastic-induced nausea and vomiting \\ Farmacoterapia actual de náuseas y vómitos inducidos por antineoplásticos
}

Recebido: 19/07/2021 | Revisado: 23/07/2021 | Aceito: 27/07/2021 | Publicado: 02/08/2021

\author{
Aline Kely Felício de Sousa Santos \\ ORCID: https://orcid.org/0000-0002-2720-3129 \\ Residência Multiprofissional em Saúde da Criança, Brasil \\ E-mail: alinekely.f@gmail.com \\ Anaís Bezerra de Gusmão \\ ORCID: https://orcid.org/0000-0001-7170-9410 \\ Universidade de Pernambuco, Brasil \\ E-mail: anaisgusmao@gmail.com \\ Lucas Nóbrega de Oliveira \\ ORCID: https://orcid.org/0000-0003-2656-6910 \\ Residência Multiprofissional em Saúde da Criança, Brasil \\ E-mail: lucasnobrega18@outlook.com \\ Richard Morrinson Couras de Carvalho \\ ORCID: https://orcid.org/0000-0002-2564-3657 \\ Residência Multiprofissional em Saúde da Criança, Brasil \\ E-mail: rich_couras@hotmail.com \\ Cibério Landim Macedo \\ ORCID: https://orcid.org/0000-0002-0824-4056 \\ Residência Multiprofissional em Saúde da Criança, Brasil \\ E-mail: ciberiolandim@hotmail.com
}

\section{Resumo}

As náuseas e vômitos induzidos por quimioterapia (NVIQ) são reações adversas à medicamentos comuns com o uso de antineoplásicos. Embora existam avanços na terapêutica profilática, o manejo de medicamentos antieméticos na prática clínica ainda tem sido um desafio a ser enfrentado. O objetivo do presente estudo foi buscar na literatura científica as principais terapias farmacológicas utilizadas nesta condição. Uma revisão sistemática foi realizada, no qual buscou-se artigos científicos indexados no PubMed, ScienseDirect e Web of Science, utilizando os seguintes descritores: chemotherapy; nausea; vomiting; drug therapy; serotonin 5-HT 3 receptor antagonists, neurokinin-1 receptor antagonista. $\mathrm{O}$ grau de emetogenicidade causado pelos agentes antineoplásicos influencia diretamente no tratamento das NVIQ, que incluem os antagonistas dos receptores de serotonina (5-HT 3 ), antagonistas dos receptores de neurocinina (NK-1). Este tratamento pode ser feito de forma individualizada, porém, caso ele não seja responsivo, é possível fazer a associação entre essas duas classes. Em casos mais complicados é indicado uma terapia tripla que é composta pela combinação de antagonistas dos receptores de 5- $\mathrm{HT}_{3}$ com antagonistas dos receptores de NK-1 e dexametasona. Portanto, a eficácia da terapia antiemética é aumentada nessa tríadequando comparados ao tratamento desses medicamentos de forma individualizada. Os antagonistas dos receptores de 5-HT 3 de primeira geração são muito eficazes, contudo, a palonosetrona, de segunda geração, é mais eficaz e seguro. Uma formulação de netupitanto e palonosetrona, quando administrado juntamente com a dexametasona, tem sido uma boa opção para o tratamento de NVIQ.

Palavras-chave: Antagonistas dos receptores 5- $\mathrm{HT}_{3}$; Antagonistas dos receptores NK-1; Corticosteroide; Náuseas e vômitos induzidos por quimioterapia.

\begin{abstract}
Chemotherapy-induced nausea and vomiting (CINV) are common adverse drug reactions with the use of anticancer drugs. Although there are advances in prophylactic therapy, the management of antiemetic drugs in clinical practice is still a challenge to be faced. The aim of this study was to search the scientific literature for the main pharmacological therapies used in this condition. A systematic review was carried out, in which scientific articles indexed in PubMed, ScienseDirect and Web of Science were searched, using the following descriptors: chemotherapy; nausea; vomiting; drug therapy; serotonin 5-HT3 receptor antagonists, neurokinin-1 receptor antagonist. The degree of emetogenicity is increased by antineoplastic agents directly in the treatment of CINV, which include serotonin (5-HT3) receptor antagonists, neurokinin (NK-1) receptor antagonists. This treatment can be done individually, however, if it is not responsive, it is possible to make the association between these two classes. In more complicated cases, triple therapy is indicated, consisting of a combination of 5-HT3 receptor antagonists with NK-1 and dexamethasone receptor antagonists. Therefore, an efficacy of antiemetic therapy is increased in this triad when compared to drug treatment
\end{abstract}


individually. First-generation 5-HT3 receptor antagonists are very effective, however, second-generation palonosetron is more effective and safer. A portion of netupitant and palonosetron, when given together with a dexamethasone, has been a good option for the treatment of CINV.

Keywords: 5- $\mathrm{HT}_{3}$ receptor antagonists; NK-1 receptor antagonists; Corticosteroid; Chemotherapy-induced nausea and vomiting.

\section{Resumen}

Las náuseas y los vómitos inducidos por la quimioterapia (NVIQ) son reacciones adversas comunes a los medicamentos con el uso de medicamentos contra el cáncer. Aunque existen avances en la terapia profiláctica, el manejo de los fármacos antieméticos en la práctica clínica sigue siendo un desafío por afrontar. El objetivo de este estudio fue buscar en la literatura científica las principales terapias farmacológicas utilizadas en esta patología. Se realizó una revisión sistemática, en la que se buscaron artículos científicos indexados en PubMed, ScienseDirect y Web of Science, utilizando los siguientes descriptores: quimioterapia; náusea; vómitos terapia de drogas; antagonistas del receptor de serotonina 5-HT3, antagonistas del receptor de neuroquinina-1. El grado de emetogenicidad causado por agentes antineoplásicos influye directamente en el tratamiento de las NVIQ, que incluyen antagonistas del receptor de serotonina (5-HT3) y antagonistas del receptor de neuroquinina (NK-1). Este tratamiento se puede realizar de forma individual, sin embargo, si no responde, es posible realizar la asociación entre estas dos clases. En casos más complicados, está indicada la terapia triple, que consiste en una combinación de antagonistas del receptor 5-HT3 con antagonistas del receptor NK-1 y dexametasona. Por tanto, la eficacia de la terapia antiemética aumenta en esta tríada en comparación con el tratamiento de estos fármacos de forma individual. Los antagonistas del receptor 5-HT3 de primera generación son muy eficaces; sin embargo, el palonosetrón de segunda generación es más eficaz y seguro. Una formulación de netupitant y palonosetron, cuando se administra junto con dexametasona, ha sido una buena opción para el tratamiento de las NVIQ.

Palabras clave: Antagonistas del receptor 5-HT 3 ; Antagonistas del receptor NK-1; Corticosteroide; Náuseas y vómitos inducidos por quimioterapia.

\section{Introdução}

Náuseas e vômitos induzidos por quimioterapia (NVIQ) são os efeitos adversos mais comuns em pacientes com câncer (Chow et al., 2020). Sendo associados a uma deterioração da qualidade de vida do paciente (Navari, 2017). Apesar do desenvolvimento de novos agentes antieméticos, o controle das náuseas e vômitos continua sendo um problema para muitos pacientes com câncer (Hendren et al., 2015; Ruhlmann \& Herrstedt, 2016). Portanto, pesquisas sobre o seu tratamento são necessárias, pois estes podem alterar a rotina do paciente e até interromper o tratamento medicamentoso, causando desidratação, perda de vitaminas, eletrólitos e minerais, além de ferimentos no tubo digestivo (esôfago), promovendo desconforto no paciente e possível descontinuação do tratamento.

O tratamento para náuseas e vômitos, a fim de reduzir os efeitos adversos do tratamento antineoplásico e uma melhor adesão à farmacoterapia, é relevante para um aumento significativo da qualidade de vida dos pacientes (Moretto et al., 2019). No Brasil, o Consenso Brasileiro de Náuseas e Vômitos em Cuidados Paliativos descreve o instrumento de avaliação de náuseas e vômitos da Associação Multinacional de Suporte no Cuidado em Câncer (MASCC) e da Sociedade Europeia de Oncologia Médica (ESMO) como protocolo a ser seguido (Simino et al., 2020). Sendo assim, a eficácia dos antieméticos deve ser melhor explorada (Andrews \& Horn, 2006).

Antieméticos são uma classe de medicamentos capazes de prevenir náuseas e vômitos. Podem ser administradas antes ou após a quimioterapia (Andrews \& Horn, 2006; Chow et al., 2020). A escolha dos antieméticos deve ser baseada no risco emético da terapia antineoplásica e experiência anterior com antieméticos, além dos fatores de risco do paciente como idade e sexo. Não foram identificados achados clínicos relevantes de interações medicamentosas entre antineoplásicos e regimes utilizando antieméticos (Chow et al., 2016).

Embora os antieméticos modernos tenham diminuído em grande parte as NVIQ, o manejo da náusea e do vômito ainda se torna desafiador (Moretto et al., 2019). Nesse contexto, a farmacoterapia abrange várias classes de antieméticos, incluindo os antagonistas dos receptores de serotonina do tipo 3 e antagonistas dos receptores de neurocinina tipo 1 (Lau et al., 
2016; Navari, 2017). Logo, o presente estudo teve como objetivo investigar na literatura nacional e internacional as principais classes de fármacos, que são mais utilizados na prevenção/tratamento desses efeitos adversos, bem como avaliar a eficácia de suas associações, através de uma análise sistemática nas bases eletrônicas de dados.

\section{Metodologia}

As revisões sistemáticas são uma forma de reunir evidências científicas atuais disponíveis em plataformas on-line que utiliza uma metodologia claramente desenhada a fim de identificar e avaliar criticamente pesquisas relevantes através da organização e análise de dados (Roever, 2017). Sendo assim, o presente estudo trata-se de uma revisão da literatura, de caráter qualitativo, elaborada conforme as instruções contidas no Preferred Reporting Items for Systematic reviews and MetaAnalyses (PRISMA).

A metodologia PRISMA, segundo Liberati et al. (2009), garante uma verificação completa e transparente de revisões sistemáticas e meta-análises elaboradas, sendo um importante recursos para melhorar os relatos obtidos. Nesta revisão, as bases eletrônicas de dados exploradas a fim de obter artigos científicos foram Sciencedirect, Web of Science e National Library of Medicine (Pubmed).

Para elaboração desta pesquisa, a busca, a seleção, a extração e a análise dos dados foram realizadas aos pares, seguida de discussão entre os autores, que elegeram as publicações mais pertinentes para integrarem o estudo. No entanto, havendo divergência entre eles, um terceiro autor foi consultado para a resolução da possível discordância.

Os critérios de inclusão escolhidos para a seleção dos materiais foram: artigos nas categorias original, revisão deliteratura, revisão sistemática e meta-análise. As publicações selecionadas foram nos idiomas português e inglês. Os descritores presentes nos títulos e/ou resumos dos materiais utilizados foram: chemotherapy; nausea; vomiting; drug therapy;serotonin 5-HT3 receptor antagonists, neurokinin-1 receptor antagonist. As publicações consideradas mais relevantes para o desenvolvimento desta revisão foram consideradas entre os anos de 2015 e 2021, sendo este o intervalo de tempo adotado.

Foram excluídas desta pesquisa as publicações consideradas sem relação com o objetivo do tema deste trabalho, os publicados nos formatos de trabalho de conclusão de curso, dissertação, tese, relato de caso, resenha e resumo de congresso, assim como em outros idiomas além do português e do inglês. A começar pela leitura dos resumos, excluiu-se aqueles que não continham informaç̃es que contemplassem o tema para esta revisão bibliográfica ou que apresentassem informações repetidas.

\section{Resultados e Discussão}

Foram encontrados 132 artigos nas bases de dados mencionada, como pode ser observado na Figura 1. Destes, 11 foram excluídos por duplicidade. Além disso, 41 não mostraram relação com o tema, e 18 não continham dados suficientes descritos. Por fim, 62 artigos foram escolhidos e introduzidos nessa revisão através de uma análise cuidadosa. 
Figura 1: Fluxograma demostrando o processo de seleção de artigos nas bases de dados utilizadas.

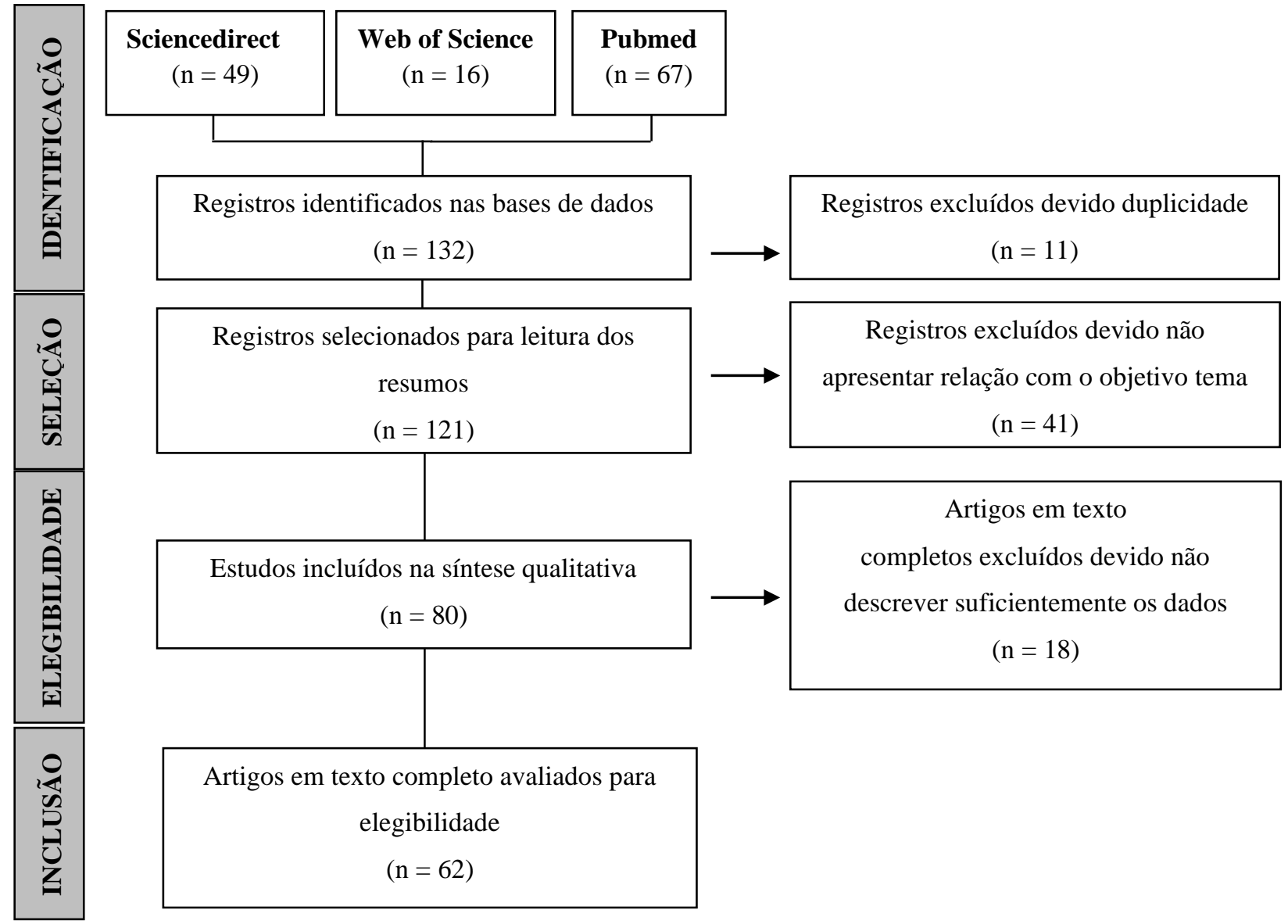

Fonte: Autores.

\section{Náuseas e vômitos induzidos por quimioterapia}

As NVIQ influenciam diretamente a saúde e a qualidade de vida dos pacientes com câncer, levando a possíveis hospitalizações e aumento dos custos na saúde pública, afetando até $40 \%$ dos pacientes em tratamentos, além de desestimulálos a continuar o tratamento antineoplásico (Aapro, 2018; Shankar et al., 2015). Alguns estudos apontam que, apesar da profilaxia antiemética, 64,4\% dos pacientes que fazem quimioterapia apresentam emetogenicidade que, se não resolvidas, podem resultar em sequelas como anorexia, distúrbios eletrolíticos, desidratação, fraqueza, perda de peso e trauma gástrico ou esofágico (Li et al., 2019; Navari, 2015).

Os agentes antineoplásicos podem ser classificados pelo seu potencial emetogênico em: mínimo (<10\%), baixo (1030\%), moderado (30-90\%) e alto (> 90\%) (Smith et al., 2020; Totadri, 2016). Antineoplásicos considerados altamente emetogênicos incluem cisplatina em altas doses, carmustina, ciclofosfamida (em doses superiores a $1500 \mathrm{~g} / \mathrm{m}^{2}$ ), dacarbazina, mecloretamina, estreptozocina e combinações de antraciclinas e fosfamida. Já a carboplatina, doxorrubicina, irinotecano, oxaliplatina e ciclofosfamida são considerados antineoplásicos moderadamente emetogênicos (Gupta et al., 2021). Quando não tratada, a quimioterapia altamente emetogênica tem uma ocorrência nos pacientes que excede até 90\% (Hashimoto et al., 2020).

Os fatores envolvidos na quimioterapia que podem aumentar as náuseas e vômitos são: sexo, idade, ansiedade, consumo de álcool, vômitos associados a gravidez ou enjoos, vômitos durante um ciclo de quimioterapia anterior e antiemético administrado (Eakin et al, 2020, Ranganath et al., 2015). As NVIQ são classificadas de acordo com o tempo em que elas podem ocorrer. A fase aguda ocorre 24 horas após a administração da quimioterapia e geralmente os sintomas são mediados 
pela atividade da serotonina, já a fase tardia ocorre entre 24 horas a 5 dias após a quimioterapia, no qual se predomina a atividade da substância P que envolve os receptores de neurocinina no sistema nervoso central (Poma \& Garcia, 2017; Smith et al., 2020; Totadri,2016).

Estudos que associam a ocorrência de NVIQ e sua gravidade mostram que podem existir alguns polimorfismos no receptor de serotonina, bem como nas enzimas que metabolizam os antieméticos, contudo pesquisas adicionais são necessárias (Candiotti et al., 2020; Singh et al., 2018). Sendo assim, biomarcadores farmacogenômicos podem se tornar um fator importante de "medicina personalizada" nos serviços prestados a pacientes com câncer trazendo impacto numa resposta profilática e terapêutica das NVIQ (Goto et al., 2016).

De acordo com algumas diretrizes internacionais como a National Comprehensive Cancer Network (NCCN), American Society of Clinical Oncology (ASCO) e Multinational Association of Supportive Care in Cancer (MASCC) / European Society for Medical Oncology (ESMO), o regime antiemético padrão inclui 3 classes de drogas: antagonistas dos receptores de serotonina $\left(5-\mathrm{HT}_{3}\right)$, antagonistas dos receptores de neurocinina 1 (NK-1) e corticosteroides. Tal regime pode ainda ser complementado com a olanzapina (antipsicótico), como pode ser observado no Quadro 1.

Quadro 1. Profilaxia de náuseas e vômitos induzidos por antineoplásicos

\begin{tabular}{|c|c|c|}
\hline \multicolumn{3}{|c|}{ Quimioterapia altamente emetogênica } \\
\hline Diretriz & Dia 1 & Dia 2,3 e 4 \\
\hline $\begin{array}{l}\text { ASCO } \\
(2017)\end{array}$ & $\begin{array}{l}\text { Antagonista do receptor NK-1 + antagonista do receptor 5-HT } 3+ \\
\text { corticosteroide (dexametasona VO ou IV) + olanzapine VO }\end{array}$ & $\begin{array}{c}\text { Aprepitanto (d2, d3) + Dexametasona VO ou IV } \\
\qquad(\mathrm{d} 2, \mathrm{~d} 3, \mathrm{~d} 4)\end{array}$ \\
\hline $\begin{array}{l}\text { MASCC/ESMO } \\
\text { (2016) }\end{array}$ & $\begin{array}{c}\text { Antagonista do receptor NK-1 + antagonista do receptor 5-HT } 3+ \\
\text { corticosteroide (dexametasona VO ou IV) }\end{array}$ & $\begin{array}{l}\text { Aprepitanto (d2, d3) + Dexametasona VO ou IV } \\
\qquad(\mathrm{d} 2, \mathrm{~d} 3, \mathrm{~d} 4)\end{array}$ \\
\hline \multirow{3}{*}{$\begin{array}{l}\text { NCCN } \\
(2018)\end{array}$} & $\begin{array}{l}\text { Opção 1: Antagonista do receptor NK-1 + antagonista do receptor 5- } \\
\qquad \mathrm{HT}_{3}+\text { corticosteroide (dexametasona VO ou IV) }\end{array}$ & $\begin{array}{c}\text { Aprepitanto VO }(\mathrm{d} 2, \mathrm{~d} 3)+\text { dexametasona VO ou } \\
\text { IV }(\mathrm{d} 2, \mathrm{~d} 3, \mathrm{~d} 4)\end{array}$ \\
\hline & $\begin{array}{c}\text { Opção 2: Olanzapine VO + palonosetrona IV + corticosteroide } \\
\text { (dexametasona VO ou IV) }\end{array}$ & Olanzapina VO (d2, d3, d4) \\
\hline & $\begin{array}{c}\text { Opção 3: Olanzapine VO + Antagonista do receptor NK-1 + } \\
\text { antagonista do receptor 5-HT } 3 \text { + corticosteroide (dexametasona VO } \\
\text { ou IV) }\end{array}$ & $\begin{array}{l}\text { Olanzapina VO (d2, d3, d4) + aprepitanto VO } \\
\qquad(\mathrm{d} 2, \\
\mathrm{d} 3)+ \text { dexametasona VO ou IV }(\mathrm{d} 2, \mathrm{~d} 3, \mathrm{~d} 4)\end{array}$ \\
\hline \multicolumn{3}{|c|}{ Quimioterapia moderadamente emetogênica } \\
\hline Diretriz & Dia 1 & Dia 2 e 3 \\
\hline $\begin{array}{l}\text { ASCO } \\
(2017)\end{array}$ & $\begin{array}{l}\text { Antagonista do receptor } 5-\mathrm{HT}_{3}+\text { corticosteroide (dexametasona VO } \\
\qquad \text { ou IV) }\end{array}$ & Dexametasona VO ou IV (d2, d3) \\
\hline $\begin{array}{l}\text { MASCC/ESMO } \\
\text { (2016) }\end{array}$ & $\begin{array}{l}\text { Antagonista do receptor } 5-\mathrm{HT}_{3}+\text { corticosteroide (dexametasona VO } \\
\qquad \text { ou IV) }\end{array}$ & Dexametasona VO ou IV (d2, d3) \\
\hline \multirow{2}{*}{$\begin{array}{l}\text { NCCN } \\
(2018)\end{array}$} & $\begin{array}{c}\text { Opção 1: Antagonista do receptor } 5-\mathrm{HT}_{3}+\text { corticosteroide } \\
\text { (dexametasona VO ou IV) }\end{array}$ & $\begin{array}{l}\text { Dexametasona VO ou IV (d2, d3) ou antagonista } \\
\text { do receptor } 5-\mathrm{HT}_{3} \text { (monoterapia) }(\mathrm{d} 2, \mathrm{~d} 3)\end{array}$ \\
\hline & Opção 2: Olanzapine VO + palonosetrona IV + corticosteroide & Olanzapina VO (d2, d3) \\
\hline
\end{tabular}




\begin{tabular}{|c|c|c|}
\hline & (dexametasona IV) & Aprepitanto VO (d2, \\
\cline { 2 - 3 } & $\begin{array}{c}\text { Opção 3: Antagonista do receptor NK-1 + antagonista do receptor 5- } \\
\mathrm{HT}_{3}+\text { corticosteroide (dexametasona VO ou IV) }\end{array}$ & $\mathrm{d} 3$ ) + dexametasona VO ou IV (d2, d3) \\
\hline
\end{tabular}

Abreviaturas: 5-HT3: serotonina; ASCO: American Society of Clinical Oncology; ESMO: European Society for Medical Oncology; IV, intravenoso; MASCC, Multinational Association for Supportive Care in Cancer; NCCN, National Comprehensive Cancer Network; NK-1: neurocinina 1; SC, subcutâneo; VO, oral.

Fonte: Adaptado de ASCO, MASCC/ESMO e NCCN.

O princípio chave está focado na prevenção da NVIQ, e não diretamente no seu tratamento (Schwartzberg, 2018). Para a quimioterapia altamente emetogênica o regime padrão é administrado, podendo seguir-se por até 4 dias caso as náuseas e vômitos não consigam ser controladas imediatamente. Para a moderadamente emetogênica uma combinação de duas drogas é suficiente (antagonistas dos receptores 5- $\mathrm{HT}_{3}$ e corticosteroides), contudo se o risco de NVQI for aumentado pode-se adicionar um antagonista dos receptores NK-1 (Chow et al., 2018; Eakin et al, 2020; Ishido et al., 2016; Navari, 2015).

A NCCN ainda recomenda que, além da emetogenicidade, sejam considerados outros fatores como vias de administração preferidas, custos e efeitos adversos dos antieméticos e adesão e fatores de riscos relacionados ao paciente. Apesar da disponibilidade de vários agentes antieméticos, a prevenção da NVIQ ainda é um desafio, sendo necessária uma melhor compreensão das diretrizes antieméticas para garantir o seu uso rotineiro na prática (Eakin et al, 2020; Smith et al., 2020).

\section{Antagonistas do receptor de serotonina}

Os receptores de $5-\mathrm{HT}_{3}$ são canais de $\mathrm{Na} / \mathrm{K}$ que podem ser encontrados na zona de gatilho quimiorreceptora e fibras aferentes do nervo vago no intestino (Kaye et al., 2017). Os antagonistas de serotonina bloqueiam seletivamente os receptores 5- $\mathrm{HT}_{3}$ pré-sinápticos no nervo vago e na área postrema, bloqueando os sinais transmitidos pelos aferentes do nervo vago, agindo como antieméticos por inibirem a estimulação periférica (Gupta et al., 2021). Os antagonistas dos receptores 5-HT 3 mais utilizados incluem a granisetrona (via oral, intravenosa, subcutânea ou transdérmica), ondansetrona (via oral ou intravenosa), palonasetrona (via intravenosa) e dolasetrona (Smith et al., 2020).

Ainda pode-se dividir os antagonistas dos receptores 5- $\mathrm{HT}_{3}$ de acordo com o seu tempo de meia-vida, potência, interação molecular com os receptores 5- $\mathrm{HT}_{3}$ e eficácia no controle de NVIQ na fase retardada, em primeira geração e segunda geração. Nos de primeira geração estão inclusos a odansetrona, dolasetrona e granisetrona. A palonosetrona é um antagonista de segunda geração que possui uma meia-vida mais longa e afinidade maior pelos receptores de serotonina, o que pode explicar uma maior duração nos seus efeitos, e consequentemente maior eficácia em NVIQ na fase retardada (Navari, 2015; Rojas \& Slusher, 2015; Zarkadas et al., 2020).

As RAM mais comum dos antagonistas dos receptores de serotonina são cefaleia, constipação, diarreia e enzimas hepáticas elevadas, já efeitos menos comuns estão associados à atividade elétrica do coração anormal (prolongamento do intervalo QT, insuficiência cardíaca congestiva, bradicardia), sendo necessário o monitoramento rotineiro dos pacientes que possuem doenças cardíacas com eletrocardiograma (Eakin et al, 2020). Efeitos adversos raros incluem a síndrome serotoninérgica, que pode resultar da associação com outras drogas serotoninérgicas (Gupta et al., 2021; Navari, 2015).

A ondansetrona foi o primeiro antagonista dessa classe aprovado pela Food and Drug Administration (FDA) para o tratamento de NVIQ (Eakin et al, 2020). Se caracteriza como um fármaco de ação curta, com meia-vida de 3 a 6 horas, que inibe seletivamente os receptores $5-\mathrm{HT}_{3}$. Portanto, deve ser administrado em cada dia de quimioterapia, pois se torna mais eficaz no tratamento de NVIQ na fase aguda do que na fase retardada (Smith et al., 2020). É um fármaco bem tolerado, no qual já foi aprovado seu uso em crianças, cujo efeitos adversos relatados são de leve a moderado, contudo, está associado com o 
risco de arritmias causada pelo prolongamento do intervalo QT podendo levar a torsade de pointes dependendo da dose e do tempo de administração (Ames \& Machovec, 2020). Em termos de custos financeiros, a ondasetrona é favorável quando comparadas aos demais antagonistas 5-HT (Gupta et al., 2021; Kaye et al., 2017).

A dolasetrona foi retirada do mercado pelo FDA devido ao risco excessivo do prolongamento do intervalo QT e aumento da duração do QRS (Ames \& Machovec,2020; Barni et al., 2016).

Granisetrona é um agente antiemético bem estabelecido na prática clínica, tem ação curta, com meia-vida de 12 horas, o que também a torna é mais eficaz na fase aguda do que na tardia (Navari, 2015). Estudos demonstram que não houve nenhum efeito significativo no prolongamento do QT em pacientes tratados com a granisetrona (Barni et al., 2016). Recentemente foi desenvolvida uma formulação subcutânea de liberação prolongada para fornecer níveis terapêuticos sustentados por até 5 dias após uma única injeção, que pode contornar essa problemática e atuar na fase tardia, melhorando a adesão ao paciente dos antieméticos (Smith et al., 2020). Contudo, essa formulação não pode ser utilizada em intervalos menores que uma semana. Além disso, pela via transdérmica, a granisetrona é aplicada entre 24 a 48 horas antes da primeira dose de quimioterapia, sendo eficaz por 7 dias (Eakin et al, 2020). Na população pediátrica, a granisetrona já foi adicionada como opção terapêutica (Ames \& Machovec, 2020).

A palonosetrona é caracterizada por sua meia-vida mais longa, em torno de 40 horas, e maior eficácia. Isso está relacionado ao fato de que a sua ligação ao receptor se faz de forma distinta dos demais antagonistas $5-\mathrm{HT}_{3}$, no qual o perfil de ligação inclui interações alostéricas 100 vezes maior do que os antagonistas de primeira geração, bem como a capacidade de internalizar o receptor. Dessa forma, ela tem uma maior vantagem sobre os outros antagonistas 5-HT 3 , com efeitos terapêuticos que podem durar até 72 horas (Kaye et al., 2017; Rojas \& Slusher, 2015). Além disso, estudos confirmaram um ótimo perfil de segurança cardiovascular da palonosetrona, no qual não houve evidência de alterações no intervalo QT, sendo configurada como droga de escolha para pacientes cardiopatas (Barni et al., 2016). Embora a palanosetrona seja considerada superior aos antagonistas de primeira geração, ela não está disponível para uso ambulatorial (Eakin $e t$ al, 2020). Não requer ajuste de dose para pacientes geriátrico, com insuficiência renal e hepática, bem como não é indicado para menores de 18 anos, pois sua segurança e eficácia ainda não foi estabelecida nesse grupo (Ames \& Machovec, 2020).

Os representantes dos antagonistas do receptor de $5-\mathrm{HT}_{3}$ se encontram na Tabela 1, na qual pode ser observado as principais RAM e o tempo de meia-vida.

Tabela 1. Representantes dos antagonistas do receptor de $5-\mathrm{HT}_{3}$

\begin{tabular}{ccc}
\hline Medicamentos & RAM & Meia-vida \\
\hline Ondansetrona & Arritmias e prolongamento do QT & $3-6$ horas \\
Dolasetrona* & Prolongamento do QT e aumento de duração do QRS & - \\
Granisetrona & Sem RAM significativamente importantes & 12 horas \\
Palonosetrona & Sem RAM significativamente importantes & 40 horas \\
\hline
\end{tabular}

*Retirado do mercado pelo FDA

Fonte: Navari (2015); Rojas \& Slusher (2015); Zarkadas et al. (2020).

\section{Antagonistas do receptor de neurocinina}

Os receptores NK-1 podem ser encontrados tanto no núcleo do trato solitário como na área postrema (Kaye et al., 2017). Dessa forma, antagonistas dos receptores NK-1 atuam bloqueando competitivamente a ligação da substância P a este 
receptor (Ahmed et al., 2018; Gupta et al., 2021). Portanto, esses antagonistas são recomendados em pacientes que recebem quimioterapia altamente ou moderadamente emetogênica (Eakin et al, 2020; Zhang et al., 2016). Essa classe de antieméticos inclui o aprepitanto, fosaprepitanto, netupitanto, fosnetupitanto, rolapitanto e tradipitanto (Smith et al., 2020). Os efeitos adversos mais comuns incluem dor de cabeça, fadiga, diarreia, soluços, anorexia, constipação, alopecia e aumento das enzimas hepáticas (Gupta et al., 2021; Rojas \& Slusher, 2015).

Aprepitanto, primeiro agente disponível dessa classe, tem meia-vida de 9 a 13 horas, e quando combinado com a dexametasona demonstrou ótimos efeitos antieméticos na quimioterapia com antraciclinase na quimioterapia de moderada emetogenecidade (Koth \& Kolesar, 2017; Li et al., 2019; Llombart-Cussac et al., 2016; Yahata et al., 2016). Fornece um mecanismo complementar para a prevenção de NVIQ quando combinado com os antagonistas de 5-HT3 (Koth \& Kolesar, 2017). Está disponível em formulações orais (cápsulas e suspensão oral) (Eakin et al, 2020). Estudos clínicos mostram que o medicamento não possui efeitos adversos cardiovasculares e é seguro e bem tolerado em crianças o que determina sua indicação para NVIQ em crianças com idade superior a 6 meses na forma de suspensão oral (Ames \& Machovec, 2020; Barni et al., 2016).

Fosaprepitanto é um pró-fármaco do aprepitanto, que é convertido rapidamente em aprepitanto após a administração endovenosa por enzimas fosfatases, estando disponível em formulações parenterais e pode ser utilizado em crianças com idade de 6 meses a 12 ano (Ahmed et al., 2018; Ames \& Machovec, 2020; Eakin et al, 2020). Possui meia-vida similar a do aprepitando, entre 9 a 13 horas (Lasseter et al., 2007). Mostrou-se tão eficaz quando o aprepitando quando combinado com a ondansentrona e dexametasona no controle das NVIQ (Barni et al., 2016).

Netupitanto está disponível em comprimidos apenas em combinação de dose fixa com a palonosetrona (NEPA) (Eakin et al, 2020). O netupitanto consegue atravessar a barreira hematoencefálica, sendo capaz de bloquear efetivamente a substância P na indução das náuseas e vômitos. Outrossim, este fármaco possui uma meia-vida de aproximadamente 90 horas, ou seja, bem superior ao do aprepitanto, o que permite uma menor frequência de administração e suas RAM mais importantes são cefaleia, constipação e fadiga (Rowbottom et al., 2016).

Rolapitanto, é um antagonista dos receptores NK-1 de longa duração, com meia-vida de aproximadamente 135 a 231 horas e boa penetração no sistema nervoso central, o que favorece o seu uso na fase tardia da NVIQ e com esquemas de administração distantes (Ahmed et al., 2018; Koth \& Kolesar, 2017; Rapoport et al., 2016; Wang et al., 2019). O rolapitanto é disponibilizado em formulações orais (Eakin et al, 2020). Contudo, seu uso foi suspenso em 2018 devido a questões de segurança (Smith et al., 2020).

Um estudo recente, mostrou que o tradipitanto é um potente inibidor dos receptores NK-1, com a hipótese de atuar de forma central (centro do vômito) e periférica (músculo liso do intestino), sendo bem tolerado e com efeitos adverso semelhantes ao grupo placebo (Carlin et al., 2021). No entanto, mais estudos devem ser realizados.

As RAM e o tempo de meia-vida dos antagonistas do receptor NK-1podem ser observados na Tabela 2. 
Tabela 2. Representantes dos antagonistas do receptor de $5-\mathrm{HT}_{3}$

\begin{tabular}{ccc}
\hline Medicamentos & RAM & Meia-vida \\
\hline Aprepitanto & Sem RAM significativamente importantes & $9-13$ horas \\
Fosaprepitanto* & Sem RAM significativamente importantes & $9-13$ horas \\
Netupitanto** & Cefaleia, constipação e fadiga & 90 horas \\
Rolapitanto & Dores de cabeça, prisão de ventre, sensação de cansaço, & 180 horas \\
& diminuição do apetite, anemia e tontura. & - \\
\hline
\end{tabular}

*Pró-fármaco do aprepitanto

**Só está disponível em combinação com a palonossetrona

Fonte: Rowbottom et al. (2016); Lasseter et al. (2007); Wang et al. (2019).

\section{Corticosteroides}

A dexametasona é um corticosteroide de ação prolongada, que age através da inibição da síntese de prostaglandinas no hipotálamo (Totadri, 2016). Quando administrada para a prevenção de NVIQ ela foi associada a menos efeitos adversos (Raghunath et al., 2020). Possui início de ação rápida (via endovenosa) com efeito de duração de até 72 horas (Kaye et al., 2017). É importante ressaltar que o uso de dexametasona em pacientes com câncer deve ser monitorado, pois ela está associada a síndrome de lise tumoral no qual pode ocorrer distúrbios metabólicos desastrosos devido à destruição maciça das células malignas e liberação do seu conteúdo no espaço extracelular (Ames \& Machovec, 2020). Os corticosteroides têm diversos efeitos adversos, como insônia, hiperglicemia, redução da densidade mineral óssea, imunossupressão e aumento do riscode infecções invasivas, portanto devem ser usados com cautela (Aldea et al, 2020; Minatogawa et al., 2020; Totadri, 2016).

Combinação dos antagonistas do receptor de serotonina com antagonistas do receptor de neurocinina elou dexametasona

Apesar do uso combinado de classes de antieméticos a incidência de NVIQ em pacientes submetidos a quimioterapia alta ou moderadamente emetogênica permanece alto (61\%) (Ahmed et al., 2018). Na quimioterapia moderadamente emetogênica (aguda ou tardia) é comum as prescrições que contenham granisetrona (via subcutânea), palonossetron (via intravenosa) ou combinação de palonosetrona e netupitanto (via oral). Contudo, na quimioterapia altamente emetogênica (aguda ou retardada) as prescrições incluem a palonosetrona e netupitanto (via oral e intravenosa) para prevenir náuseas e vômitos (Osaki et al., 2019). Já na quimioterapia baseada em ciclofosfamida o mais indicado é o uso da palonosetrona e netupitanto (via oral) e granisetrona (via subcutânea) (Caputo et al., 2020).

A palonosetrona está disponível em combinação com netupitanto e com o fosnetupitanto (antagonistas NK-1), via oral e intravenosa, respectivamente. Palonosetrona não é indicada para prevenção de náuseas e vômitos induzidos por quimioterapia na fase retardada após a quimioterapia altamente emetogênica (Park et al., 2019). Contudo quando é administrada juntamente com a dexametasona houve uma melhora repostas em ambas as fases (aguda e tardia) da quimioterapia altamente emetogênica (Gupta et al., 2021). Outro estudo demonstra que a combinação de NEPA (300 mg + 0,5 mg) com dexametasona é uma boa opção para o tratamento de NVIQ altamente emetogênica (Clark-Snow et al., 2018; Navari et al., 2021).

A combinação de um antagonista dos receptores 5- $\mathrm{HT}_{3}$, antagonistas dos receptores NK-1 e dexametasona para controle da NVIQ é amplamente discutida na literatura (Hayashi et al., 2021; Isoda et al., 2017; Janicki, 2016; Kono et al., 2018; Natale et al., 2016; Rowbottom et al., 2016; Schwartzberg et al., 2018). Um estudo randomizado realizado em pacientes 
com câncer de mama e tratados com quimioterapia combinada de antraciclina com ciclofosfamida demonstrou que o uso do aprepitanto em conjunto com a tropisetrona e dexametasona controlaram as NVIQ, melhorando a qualidade de vida dos pacientes (Li et al., 2019).

Outro fator preponderante que explica a combinação de algumas classes diferentes de antieméticos é o fato de que elas atuam em receptores diferentes o que proporciona um sinergismo terapêutico na prevenção da NVIQ, além de possíveis diminuições nas doses utilizadas (Chow et al., 2018; Rowbottom et al., 2016). A utilização de NEPA apresenta mais de 85\% de eficácia clínica, alta penetração no sistema nervoso central, boa tolerabilidade e efetivo no tratamento profilático agudo e tardio de NVIQ (Rojas \& Slusher, 2015; Zhong et al., 2017). O perfil de segurança cardíaca do NEPA foi investigado, e não foram observadas diferenças significativas em parâmetros cardíacos avaliados em comparação com o placebo, mesmo em doses supraterapêuticas (Barni et al., 2016). A combinação de NEPA controlam melhor a NVIQ nas fases agudas e tardias quando comparados a outros regimes de antagonistas dos receptores de 5-HT3 (Aapro et al., 2017; Anzai et al., 2020; Chang et al., 2020; Keating, 2015; Rojas \& Slusher, 2015; Schwartzberg et al., 2019).

Contudo, estudos clínicos que avaliaram o efeito do NEPA na náusea crônica em pacientes com câncer, mostraram que não houve diferenças significativas entre o grupo que recebeu NEPA e o que recebeu placebo (Hui et al., 2021; Navari, 2017).

A terapia antiemética também pode ser realizada com a administração de aprepitanto e palonosetrona, no qual se observou um benefício clínico ao controle das NVIQ (Bubalo et al., 2018; Di Renzo et al, 2020, Ioroi et al., 2018; Sugimori et al., 2017; Wu et al., 2018). Estudos recentes mostram que a profilaxia de NVIQ em pacientes pediátricos é feito através de um regime triplo que utiliza um antagonista 5-HT3, dexametasona e um antagonista NK-1, contudo, é difícil determinar os benefícios da adição de corticosteroides no tratamento da NVIQ nesses pacientes devido aos vários estudos heterogêneos existentes (Gupta et al., 2021; Sherani et al., 2019,).

Ramosetrona, em combinação com aprepitanto e dexametasona, se mostrou tanto eficaz quanto seguro quando comparado com a palonosetrona em combinação com aprepitanto e dexametasona (Kang et al., 2020)

Estudos de fase 2 para o fosaprepitanto e de fase 3 para o rolapitanto demonstram um controle melhorado de NVIQ nas fases tardia e geral quando estes fármacos são adicionados a um regime padrão de antagonista de receptor 5 - $\mathrm{HT}_{3}$ (Kumagai et al., 2018; Ruhlmann et al., 2016; Rojas \& Slusher, 2015). A esse regime também foi adicionado a dexametasona, demonstrando eficácia, segurança e tolerabilidade dessa tríade (Ahmed et al., 2018; Schwartzberg et al., 2015).

\section{Conclusão}

Ter um controle efetivo das náuseas e vômitos em pacientes com câncer que fazem o uso de antineoplásicos é um fator indispensável para que haja maior adesão ao tratamento. Nesse sentido, a escolha do medicamento antiemético é fundamental e depende de diversos fatores, incluindo o tipo de antineoplásico, tipo de emetogenicidade, condições do sistema de saúde, configuração socioeconômica do paciente e disposição deste para seguir as instruções terapêuticas. As opções farmacológicas mais recentes incluem os antagonistas dos receptores $5-\mathrm{HT}_{3}$, com ênfase para a palonosetrona devido aos resultados clínicos mais eficazes, com menos efeitos adversos e tempo de meia-vida mais longo. Os antagonistas dos receptores de NK-1 também são utilizados na terapia antiemética, porém são mais eficazes quando combinados com os antagonistas dos receptores 5- $\mathrm{HT}_{3}$, o aprepitanto e fosaprepitanto possuem menos RAM que os demais desta classe. As combinações medicamentosas entre essas classes de medicamentos com a dexametasona permite uma ótima segurança e eficácia no manejo das NVIQ de difíceis controles.

Por fim, apesar dos vários estudos abordados, é importante o aprofundamento de estudos, clínicos e não-clínicos, 
sobre os antieméticos mais recentes que estão descritos na literatura, bem como a realização de novas pesquisas a fim de serem descobertos novas classes farmacológicas de antiemético que sejam mais eficazes e com poucas reações adversas, para que o bem estar físico e psicológico dos pacientes sejam atingidos, proporcionando uma adesão ao tratamento e minimizando os desconfortos causados pela terapia com antineoplásicos.

\section{Referências}

Aapro M. (2018). CINV: still troubling patients after all these years. Supportive care in cancer: official journal of the Multinational Association of Supportive Care in Cancer, 26(1), 5-9.

Aapro, M., Karthaus, M., Schwartzberg, L., Bondarenko, I., Sarosiek, T., Oprean, C., Cardona-Huerta, S., Hansen, V., Rossi, G., Rizzi, G., Borroni, M. E., \& Rugo, H. (2017). NEPA, a fixed oral combination of netupitant and palonosetron, improves control of chemotherapy-induced nausea and vomiting (CINV) over multiple cycles of chemotherapy: results of a randomized, double-blind, phase 3 trial versus oral palonosetron. Supportive care in cancer: official journal of the Multinational Association of Supportive Care in Cancer, 25(4), 1127-1135.

Ahmed, H., Hammad, A. M., Abushouk, A. I., Zidan, M., Salem, M., Negida, A., \& Abdel-Daim, M. M. (2018). Meta-analysis of safety and efficacy of rolapitant, NK-1 receptor antagonist for prevention of chemotherapy-induced nausea and vomiting. Current problems in cancer, 42 (2), $241-255$.

Aldea, M., Orillard, E., Mansi, L., Marabelle, A., Scotte, F., Lambotte, O., \& Michot, J. M. (2020). How to manage patients with corticosteroids in oncology in the era of immunotherapy?. European journal of cancer (Oxford, England: 1990), 141, 239-251.

Ames, W. A., \& Machovec, K. (2020). An update on the management of PONV in a pediatric patient. Best practice \& research clinical anaesthesiology, 34(4), 749-758.

Andrews, P. L., \& Horn, C. C. (2006). Signals for nausea and emesis: Implications for models of upper gastrointestinal diseases. Autonomic neuroscience: basic \& clinical, $125(1-2), 100-115$.

Anzai, Y., Kawahara, F., Kouuchi, A., Watanabe, T., Saito-Inoue, K., Ishida, Y., Saito, N., \& Kimoto, S. (2020). Gan to kagaku ryoho. Cancer \& chemotherapy, 47(9), 1325-1330.

Associação Brasileira de Cuidados Paliativos (2011). Consenso Brasileiro de Náuseas e Vômitos em Cuidados Paliativos. Rev Bras Cuid Paliat. 3(2), 3-25.

Barni, S., Petrelli, F., \& Cabiddu, M. (2016). Cardiotoxicity of antiemetic drugs in oncology: An overview of the current state of the art. Critical reviews in oncology/hematology, 102, 125-134.

Bubalo, J. S., Herrington, J. D., Takemoto, M., Willman, P., Edwards, M. S., Williams, C., Fisher, A., Palumbo, A., Chen, E., Blanke, C., \& Lopez, C. D. (2018). Phase II open label pilot trial of aprepitant and palonosetron for the prevention of chemotherapy-induced nausea and vomiting (CINV) in patients receiving moderately emetogenic FOLFOX chemotherapy for the treatment of colorectal cancer. Supportive care in cancer: official journal of the Multinational Association of Supportive Care in Cancer, 26(4), 1273-1279.

Caputo, R., Cazzaniga, M. E., Sbrana, A., Torrisi, R., Paris, I., Giordano, M., Montesarchio, V., Guarneri, V., Amaducci, L., Bilancia, D., Cilenti, G., Fabi, A., Collovà, E., Schirone, A., Bonizzoni, E., Celio, L., De Placido, S., \& De Laurentiis, M. (2020). Netupitant/palonosetron (NEPA) and dexamethasone for prevention of emesis in breast cancer patients receiving adjuvant anthracycline plus cyclophosphamide: a multi-cycle, phase II study. BMC cancer, $20(1), 232$.

Carlin, J. L., Lieberman, V. R., Dahal, A., Keefe, M. S., Xiao, C., Birznieks, G., Abell, T. L., Lembo, A., Parkman, H. P., \& Polymeropoulos, M. H. (2021). Efficacy and Safety of Tradipitant in Patients With Diabetic and Idiopathic Gastroparesis in a Randomized, Placebo-Controlled Trial. Gastroenterology, 160(1), 76-87. e4.

Candiotti, K., Shrestha, C., \& Silva Ceschim, M. R. (2020). Is there a place for genetics in the management of PONV?. Best practice \& research. Clinical anaesthesiology, 34(4), 713-720.

Chang, J., Chen, G., Wang, D., Wang, G., Lu, S., Feng, J., Li, W., Li, P., Lanzarotti, C., Chessari, S., \& Zhang, L. (2020). Efficacy of NEPA, a fixed antiemetic combination of netupitant and palonosetron, vs a 3-day aprepitant regimen for prevention of chemotherapy-induced nausea and vomiting (CINV) in Chinese patients receiving highly emetogenic chemotherapy (HEC) in a randomized Phase 3 study. Cancer medicine, 9(14), 5134-5142.

Chow, R., Chiu, L., Navari, R., Passik, S., Chiu, N., Popovic, M., Lam, H., Pasetka, M., Chow, E., \& DeAngelis, C. (2016). Efficacy and safety of olanzapine for the prophylaxis of chemotherapy-induced nausea and vomiting (CINV) as reported in phase I and II studies: a systematic review. Supportive care in cancer: official journal of the Multinational Association of Supportive Care in Cancer, 24(2), 1001-1008.

Chow, R., Tsao, M., Chiu, L., Popovic, M., Milakovic, M., Lam, H., \& DeAngelis, C. (2018). Efficacy of the combination neurokinin-1 receptor antagonist, palonosetron, and dexamethasone compared to others for the prophylaxis of chemotherapy-induced nausea and vomiting: a systematic review and metaanalysis of randomized controlled trials. Annals of palliative medicine, 7(2), 221-233.

Chow, R., Valdez, C., Chow, N., Zhang, D., Im, J., Sodhi, E., \& Lock, M. (2020). Oral cannabinoid for the prophylaxis of chemotherapy-induced nausea and vomiting-a systematic review and meta-analysis. Supportive care in cancer: official journal of the Multinational Association of Supportive Care in Cancer, $28(5), 2095-2103$.

Clark-Snow, R. A., Vidall, C., Börjeson, S., \& Jahn, P. (2018). Fixed Combination Antiemetic: A Literature Review on Prevention of Chemotherapy-Induced Nausea and Vomiting Using Netupitant/Palonosetron. Clinical journal of oncology nursing, 22(2), E52-E63. 
Di Renzo, N., Melillo, L., Porretto, F., Dargenio, M., Pavone, V., Pastore, D., Mazza, P., Mannina, D., Merenda, A., Cascavilla, N., Greco, G., Matera, R., Bonizzoni, E., Celio, L., \& Musso, M. (2020). Every-other-day palonosetron plus aprepitant for prevention of emesis following induction chemotherapy for acute myeloid leukemia: A randomized, controlled study from the "Rete Ematologica Pugliese". Cancer medicine, 9(1), 170-178.

Eakin, C. M., Norton, T. J., Monk, B. J., \& Chase, D. M. (2020). Management of nausea and vomiting from poly(ADP-ribose) polymerase inhibitor therapy for advanced ovarian cancer. Gynecologic oncology, 159(2), 581-587.

Goto, A., Kotani, H., Miyazaki, M., Yamada, K., Ishikawa, K., Shimoyama, Y., Niwa, T., Hasegawa, Y., \& Noda, Y. (2016). Genotype frequencies for polymorphisms related to chemotherapy-induced nausea and vomiting in a Japanese population. Journal of pharmaceutical health care and sciences, 2 , 16.

Gupta, K., Walton, R., \& Kataria, S. P. (2021). Chemotherapy-Induced Nausea and Vomiting: Pathogenesis, Recommendations, and New Trends. Cancer treatment and research communications, 26, 100278.

Hashimoto, H., Abe, M., Tokuyama, O., Mizutani, H., Uchitomi, Y., Yamaguchi, T., Hoshina, Y., Sakata, Y., Takahashi, T. Y., Nakashima, K., Nakao, M., Takei, D., Zenda, S., Mizukami, K., Iwasa, S., Sakurai, M., Yamamoto, N., \& Ohe, Y. (2020). Olanzapine 5 mg plus standard antiemetic therapy for the prevention of chemotherapy-induced nausea and vomiting (J-FORCE): a multicentre, randomised, double-blind, placebo-controlled, phase 3 trial. The Lancet. Oncology, 21(2), 242-249.

Hayashi, T., Shimokawa, M., Matsuo, K., Nishimura, J., Iihara, H., Nakano, T., \& Egawa, T. (2021). 5HT3 RA plus dexamethasone plus aprepitant for controlling delayed chemotherapy-induced nausea and vomiting in colorectal cancer. Cancer science, 112(2), 744-750.

Hendren, G., Aponte-Feliciano, A., \& Kovac, A. (2015). Safety and efficacy of commonly used antiemetics. Expert opinion on drug metabolism \& toxicology, 11(11), 1753-1767.

Hui, D., Puac, V., Shelal, Z., Liu, D., Maddi, R., Kaseb, A., Javle, M., Overman, M., Yennurajalingam, S., Gallagher, C., \& Bruera, E. (2021). Fixed-Dose Netupitant and Palonosetron for Chronic Nausea in Cancer Patients: A Double-Blind, Placebo Run-in Pilot Randomized Clinical Trial. Journal of pain and symptom management, 62(2), 223-232.

Ioroi, T., Furukawa, J., Kume, M., Hirata, S., Utsubo, Y., Mizuta, N., Miyake, H., Fujisawa, M., \& Hirai, M. (2018). Phase II study of palonosetron, aprepitant and dexamethasone to prevent nausea and vomiting induced by multiple-day emetogenic chemotherapy. Supportive care in cancer: official journal of the Multinational Association of Supportive Care in Cancer, 26(5), 1419-1423.

Ishido, K., Higuchi, K., Azuma, M., Sasaki, T., Tanabe, S., Katada, C., Yano, T., Wada, T., Koizumi, W., \& Kitasato Digestive Disease \& Oncology Group (2016). Aprepitant, granisetron, and dexamethasone versus palonosetron and dexamethasone for prophylaxis of cisplatin-induced nausea and vomiting in patients with upper gastrointestinal cancer: a randomized crossover phase II trial (KDOG 1002). Anti-cancer drugs, 27(9), 884-890.

Isoda, A., Saito, R., Komatsu, F., Negishi, Y., Oosawa, N., Ishikawa, T., Miyazawa, Y., Matsumoto, M., Sawamura, M., \& Manaka, A. (2017). Palonosetron, aprepitant, and dexamethasone for prevention of nausea and vomiting after high-dose melphalan in autologous transplantation for multiple myeloma: A phase II study. International journal of hematology, 105(4), 478-484.

Janicki P. K. (2016). Management of acute and delayed chemotherapy-induced nausea and vomiting: role of netupitant-palonosetron combination. Therapeutics and clinical risk management, 12, 693-699.

Kang, J. H., Kwon, J. H., Lee, Y. G., Park, K. U., An, H. J., Sohn, J., Seol, Y. M., Lee, H., Yun, H. J., Ahn, J. S., Yang, J. H., Song, H., Koo, D. H., Kim, J. Y., Kim, G. M., \& Kim, H. J. (2020). Ramosetron versus Palonosetron in Combination with Aprepitant and Dexamethasone for the Control of HighlyEmetogenic Chemotherapy-Induced Nausea and Vomiting. Cancer research and treatment, 52(3), 907-916.

Kaye, A. D., Cornett, E. M., Chalabi, J., Naim, N. Z., Novitch, M. B., Creel, J. B., Jhita, P., Trang, T. N., Paetzold, J. R., Darensburg, N., Beakley, B. D., \& Urman, R. D. (2017). Pharmacology of Antiemetics: Update and Current Considerations in Anesthesia Practice. Anesthesiology clinics, 35(2), e41-e54.

Keating G. M. (2015). Netupitant/Palonosetron: A Review in the Prevention of Chemotherapy-Induced Nausea and Vomiting. Drugs, 75(18), $2131-2141$.

Kono, T., Ueda, T., Takumida, M., Furuie, H., Hamamoto, T., Takeno, S., \& Hirakawa, K. (2018). Low-dose dexamethasone with fosaprepitant and palonosetron to prevent cisplatin-induced nausea and vomiting in head and neck cancer patients. Acta oto-laryngologica, 138(10), 921-925.

Koth, S. M., \& Kolesar, J. (2017). New options and controversies in the management of chemotherapy-induced nausea and vomiting. American journal of health-system pharmacy: AJHP: official journal of the American Society of Health-System Pharmacists, 74(11), 812-819.

Kumagai, H., Kusaba, H., Yamanaka, T., Nio, K., Inadomi, K., Takayoshi, K., Ito, M., Tamura, S., Makiyama, A., Makiyama, C., Hirano, G., Shibata, Y., Shirakawa, T., Mitsugi, K., Ariyama, H., Esaki, T., Akashi, K., \& Baba, E. (2018). A phase 2 study of fosaprepitant combined with high-dose dexamethasone for Japanese cancer patients receiving highly emetogenic chemotherapy. Medicine, 97(25), e11042.

Lasseter, K. C., Gambale, J., Jin, B., Bergman, A., Constanzer, M., Dru, J., Han, T. H., Majumdar, A., Evans, J. K., \& Murphy, M. G. (2007). Tolerability of fosaprepitant and bioequivalency to aprepitant in healthy subjects. Journal of clinical pharmacology, 47(7), 834-840.

Lau, T. K., Yip, C. H., \& Yeo, W. (2016). State of the Art Antiemetic Therapy for Cancer Patients. Current oncology reports, 18(1), 2.

Li, Q., Wu, Y., Wang, W., Deng, S., Jiang, C., Chen, F., Zhao, J., Li, H., Bai, X., Hou, J., Da, L., Zhao, L., Gao, J., \& Jin, G. (2019). Effectiveness and safety of combined neurokinin-1 antagonist aprepitant treatment for multiple-day anthracycline-induced nausea and vomiting. Current problems in cancer, 43(6), 100462 .

Liberati, A., Altman, D. G., Tetzlaff, J., Mulrow, C., Gøtzsche, P. C., Ioannidis, J. P., Clarke, M., Devereaux, P. J., Kleijnen, J., \& Moher, D. (2009). The PRISMA statement for reporting systematic reviews and meta-analyses of studies that evaluate health care interventions: explanation and elaboration. $P L o S$ Medicine, 6(7), e1000100. 
Llombart-Cussac, A., Ramos, M., Dalmau, E., García-Saenz, J. A., González-Farré, X., Murillo, L., Calvo, L., Morales, S., Carañana, V., González, A., Fernández-Morales, L. A., Moreno, F., Casas, M. I., Angulo, M., Cámara, M. C., Garcia-Mace, A. I., Carrasco, E., \& Jara-Sánchez, C. (2016). Incidence of chemotherapy-induced nausea and vomiting associated with docetaxel and cyclophosphamide in early breast cancer patients and aprepitant efficacy as salvage therapy. Results from the Spanish Breast Cancer Group/2009-02 study. European journal of cancer (Oxford, England: 1990), 58, 122-129.

Minatogawa, H., Izawa, N., Kawaguchi, T., Miyaji, T., Shimomura, K., Kazunori, H., Iihara, H., Ohno, Y., Inada, Y., Arioka, H., Morita, H., Hida, N., Sugawara, M., Katada, C., Nawata, S., Ishida, H., Tsuboya, A., Tsuda, T., Yamaguchi, T., \& Nakajima, T. E. (2020). Study protocol for SPARED trial: randomised non-inferiority phase III trial comparing dexamethasone on day 1 with dexamethasone on days 1-4, combined with neurokinin-1 receptor antagonist, palonosetron and olanzapine $(5 \mathrm{mg})$ in patients receiving cisplatin-based chemotherapy. BMJ open, 10(12), e041737.

Moretto I.G., Contim C. L. V., Espírito Santo, F. H. (2019) Acompanhamento por telefone como intervenção de enfermagem a pacientes em quimioterapia ambulatorial: revisão integrativa. Rev Gaúcha Enferm. 40:e20190039.

Natale, J. J., Spinelli, T., Calcagnile, S., Lanzarotti, C., Rossi, G., Cox, D., \& Kashef, K. (2016). Drug-drug interaction profile of components of a fixed combination of netupitant and palonosetron: Review of clinical data. Journal of oncology pharmacy practice: official publication of the International Society of Oncology Pharmacy Practitioners, 22(3), 485-495.

Navari R. M. (2015). 5-HT3 receptors as important mediators of nausea and vomiting due to chemotherapy. Biochimica et biophysica acta, 1848(10 Pt B), $2738-2746$

Navari R. M. (2017). Management of Chemotherapy-Induced Nausea and Vomiting in Pediatric Patients. Paediatric drugs, 19(3), $213-222$.

Navari, R. M., Binder, G., Bonizzoni, E., Clark-Snow, R., Olivari, S., \& Roeland, E. J. (2021). Single-dose netupitant/palonosetron versus 3-day aprepitant for preventing chemotherapy-induced nausea and vomiting: a pooled analysis. Future oncology (London, England), 10.2217/fon-2021-0023. Advance online publication

Osaki, A., Inoue, K., Sakai, H., Yamada, K., Minato, K., Ohyanagi, F., Tokuda, Y., Ikeda, N., Kagamu, H., Kubota, K., Tamura, T., \& Saeki, T. (2019). A dose-finding randomized Phase II study of oral netupitant in combination with palonosetron $.75 \mathrm{mg}$ intravenous for the prevention of chemotherapy-induced nausea and vomiting in Japanese patients receiving highly emetogenic chemotherapy. Japanese journal of clinical oncology, 49(2), 121-129.

Park, S. H., Binder, G., Corman, S., \& Botteman, M. (2019). Budget impact of netupitant/palonosetron for the prevention of chemotherapy-induced nausea and vomiting. Journal of medical economics, 22(8), 840-847.

Poma, J. M. \& García, L. O. (2017). Protocolo diagnóstico y terapéutico de las náuseas y vómitos en el paciente oncológico. Medicine - Programa de Formación Médica Continuada Acreditado, 12(34) 2070-2075.

Ranganath, P., Einhorn, L., \& Albany, C. (2015). Management of Chemotherapy Induced Nausea and Vomiting in Patients on Multiday Cisplatin Based Combination Chemotherapy. BioMed research international, 2015, 943618.

Raghunath, A., Chandrasekara, S. D., Anthony, S. N., \& Markman, B. (2020). Duration of dexamethasone administration for the prevention of chemotherapyinduced nausea and vomiting - A systematic review and meta-analysis. Critical reviews in oncology/hematology, 152, 103012.

Rapoport, B., Schwartzberg, L., Chasen, M., Powers, D., Arora, S., Navari, R., \& Schnadig, I. (2016). Efficacy and safety of rolapitant for prevention of chemotherapy-induced nausea and vomiting over multiple cycles of moderately or highly emetogenic chemotherapy. European journal of cancer (Oxford, England: 1990), 57, 23-30.

Roever, L. (2017). Compreendendo os estudos de revisão sistemática. Rev. Soc. Bras. Clín. Méd, 15(2), 127-130.

Rojas, C., \& Slusher, B. S. (2015). Mechanisms and latest clinical studies of new NK1 receptor antagonists for chemotherapy-induced nausea and vomiting: Rolapitant and NEPA (netupitant/palonosetron). Cancer treatment reviews, 41(10), 904-913.

Rowbottom, L., McDonald, R., Turner, A., Chow, E., \& DeAngelis, C. (2016). An Overview of Radiation-Induced Nausea and Vomiting. Journal of medical imaging and radiation sciences, 47(3S), S29-S38.

Ruhlmann, C. H., Christensen, T. B., Dohn, L. H., Paludan, M., Rønnengart, E., Halekoh, U., Hilpert, F., Feyer, P., Kristensen, G., Hansen, O., Keefe, D., \& Herrstedt, J. (2016). Efficacy and safety of fosaprepitant for the prevention of nausea and emesis during 5 weeks of chemoradiotherapy for cervical cancer (the GAND-emesis study): a multinational, randomised, placebo-controlled, double-blind, phase 3 trial. The Lancet. Oncology, 17(4), 509-518.

Ruhlmann, C. H., \& Herrstedt, J. (2016). New treatments on the horizon for chemoradiotherapy-induced nausea and vomiting. Expert opinion on pharmacotherapy, 17(12), 1623-1629.

Schwartzberg L. (2018). Getting it right the first time: recent progress in optimizing antiemetic usage. Supportive care in cancer: official journal of the Multinational Association of Supportive Care in Cancer, 26(Suppl 1), 19-27.

Schwartzberg, L., Karthaus, M., Rossi, G., Rizzi, G., Borroni, M. E., Rugo, H. S., Jordan, K., \& Hansen, V. (2019). Fixed combination of oral NEPA (netupitant-palonosetron) for the prevention of acute and delayed chemotherapy-induced nausea and vomiting in patients receiving multiple cycles of chemotherapy: Efficacy data from 2 randomized, double-blind phase III studies. Cancer medicine, 8(5), 2064-2073.

Schwartzberg, L. S., McLaughlin, T., Geller, R. B., Gabrail, N. Y., \& Marks, S. M. (2018). Real-world efficacy: intravenous palonosetron three-drug regimen for chemotherapy-induced nausea and vomiting with highly emetogenic chemotherapy. Journal of comparative effectiveness research, 7(12), 1161-1170.

Schwartzberg, L. S., Modiano, M. R., Rapoport, B. L., Chasen, M. R., Gridelli, C., Urban, L., Poma, A., Arora, S., Navari, R. M., \& Schnadig, I. D. (2015). Safety and efficacy of rolapitant for prevention of chemotherapy-induced nausea and vomiting after administration of moderately emetogenic chemotherapy or anthracycline and cyclophosphamide regimens in patients with cancer: a randomised, active-controlled, double-blind, phase 3 trial. The Lancet. Oncology, 16(9), 1071-1078. 
Research, Society and Development, v. 10, n. 9, e58910918435, 2021 (CC BY 4.0) | ISSN 2525-3409 | DOI: http://dx.doi.org/10.33448/rsd-v10i9.18435

Shankar, A., Roy, S., Malik, A., Julka, P. K., \& Rath, G. K. (2015). Prevention of Chemotherapy-Induced Nausea and Vomiting in Cancer Patients. Asian Pacific journal of cancer prevention: APJCP, 16(15), 6207-6213.

Sherani, F., Boston, C., \& Mba, N. (2019). Latest Update on Prevention of Acute Chemotherapy-Induced Nausea and Vomiting in Pediatric Cancer Patients. Current oncology reports, $21(10), 89$.

Singh, K. P., Dhruva, A. A., Flowers, E., Kober, K. M., \& Miaskowski, C. (2018). A review of the literature on the relationships between genetic polymorphisms and chemotherapy-induced nausea and vomiting. Critical reviews in oncology/hematology, 121, 51-61.

Smith, C., Smith, M., Cunningham, R., \& Davis, S. (2020). Recent Advances in Antiemetics: New Formulations of 5-HT3 Receptor Antagonists in Adults. Cancer nursing, 43(4), E217-E228.

Simino, G. P. R., Reis, I. A., Acúrcio, F. A., Andrade, E. I. G., Brazil, N. M. L. \& Cherchiglia, M. L. (2020). Fatores de risco associados a náuseas e vômitos induzidos por quimioterapia antineoplásica. Rev. Saúde Pública, 54:106.

Sugimori, Y., Ota, T., Ujihira, T., Ishiguro, T., \& Ogishima, D. (2017). A phase II randomised study to evaluate the efficacy of aprepitant plus palonosetron for preventing delayed-phase CINV associated with TC therapy in gynaecological cancer. The journal of obstetrics and gynaecology research, 43(9), 1454 1459.

Totadri, S. (2016). Prophylaxis and management of antineoplastic drug induced nausea and vomiting in children with cancer. Pediatric Hematology Oncology Journal, 1, 50-55.

Yahata, H., Kobayashi, H., Sonoda, K., Shimokawa, M., Ohgami, T., Saito, T., Ogawa, S., Sakai, K., Ichinoe, A., Ueoka, Y., Hasuo, Y., Nishida, M., Masuda, S., \& Kato, K. (2016). Efficacy of aprepitant for the prevention of chemotherapy-induced nausea and vomiting with a moderately emetogenic chemotherapy regimen: a multicenter, placebo-controlled, double-blind, randomized study in patients with gynecologic cancer receiving paclitaxel and carboplatin. International journal of clinical oncology, 21(3), 491-497.

Wang, X., Zhang, Z. Y., Wang, J., Powers, D., Arora, S., Lu, S., \& Kansra, V. (2019). Pharmacokinetics, Safety, and Tolerability of Rolapitant Administered Intravenously Following Single Ascending and Multiple Ascending Doses in Healthy Subjects. Clinical pharmacology in drug development, 8(2), 160-171.

Wu, F., Lin, X., Yang, Z., Sun, Z., Zeng, F., Heng, J., Qu, J., Zeng, L., Yang, N., \& Zhang, Y. (2018). Phase III Randomized Trial of Palonosetron and Dexamethasone With or Without Aprepitant to Prevent Nausea and Vomiting Induced by Full-dose Single-day Cisplatin-based Chemotherapy in Lung Cancer. Clinical lung cancer, 19(6), e913-e918.

Zhang, Y., Yang, Y., Zhang, Z., Fang, W., Kang, S., Luo, Y., Sheng, J., Zhan, J., Hong, S., Huang, Y., Zhou, N., Zhao, H., \& Zhang, L. (2016). Neurokinin-1 Receptor Antagonist-Based Triple Regimens in Preventing Chemotherapy-Induced Nausea and Vomiting: A Network Meta-Analysis. Journal of the National Cancer Institute, 109(2), djw217.

Zarkadas, E., Zhang, H., Cai, W., Effantin, G., Perot, J., Neyton, J., Chipot, C., Schoehn, G., Dehez, F., \& Nury, H. (2020). The Binding of Palonosetron and Other Antiemetic Drugs to the Serotonin 5-HT3 Receptor. Structure (London, England: 1993), 28(10), 1131-1140.e4.

Zhong, W., Picca, A. J., Lee, A. S., \& Darmani, N. A. (2017). Ca2+ signaling and emesis: Recent progress and new perspectives. Autonomic neuroscience: basic \& clinical, 202, 18-27. 